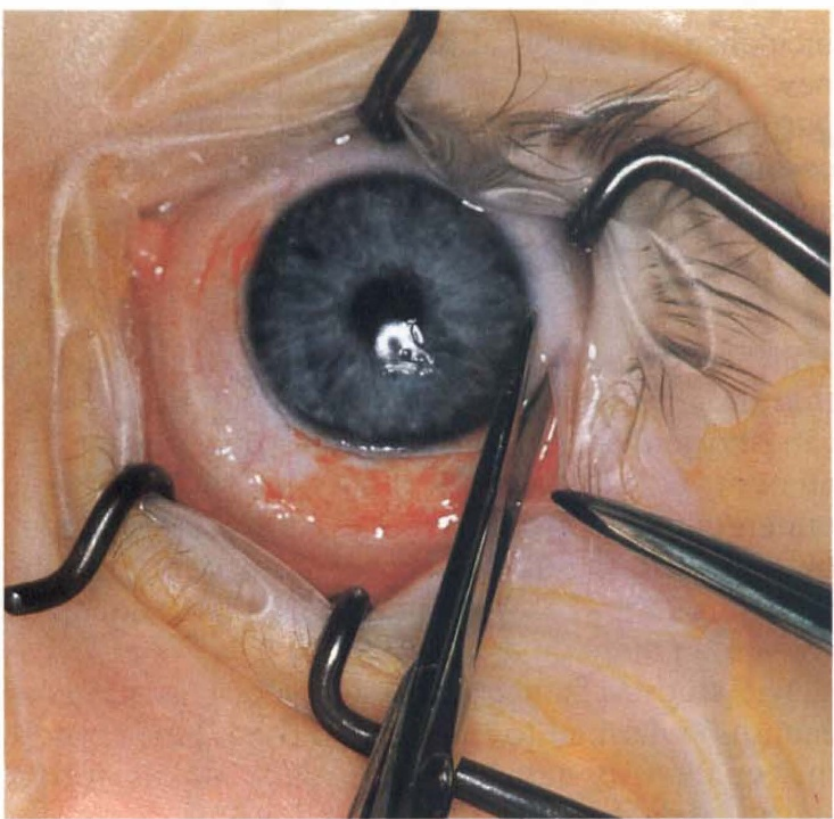

(a)

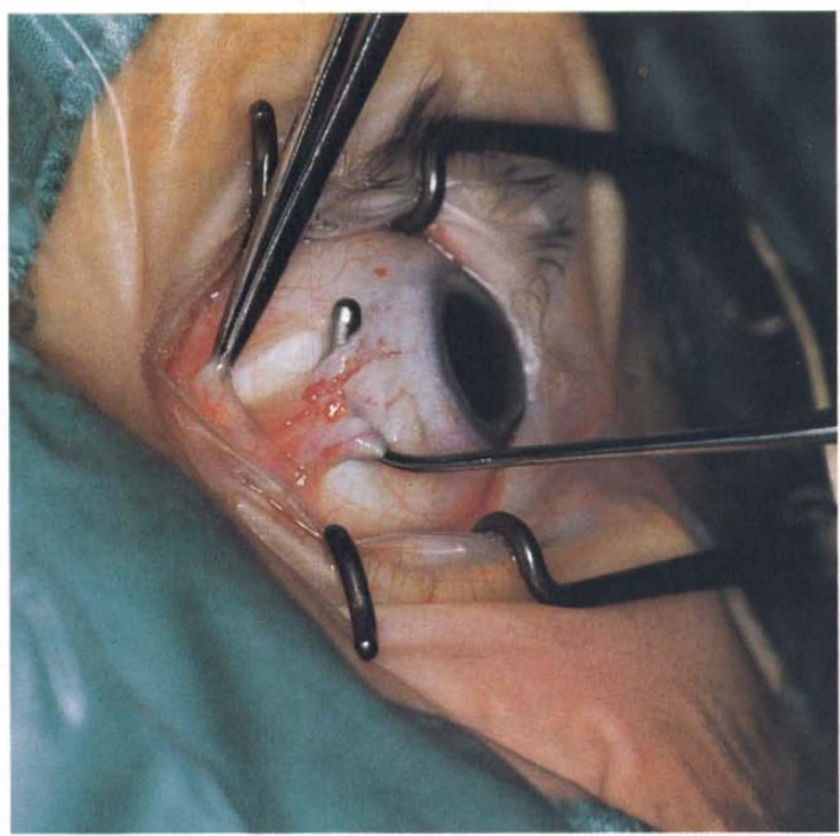

(c)

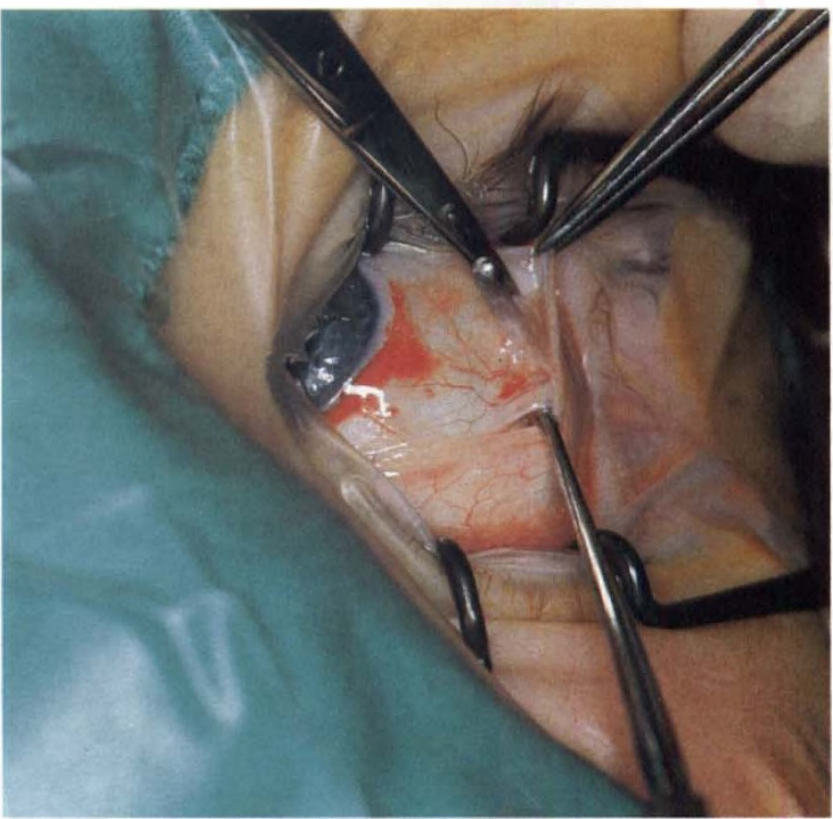

(b)

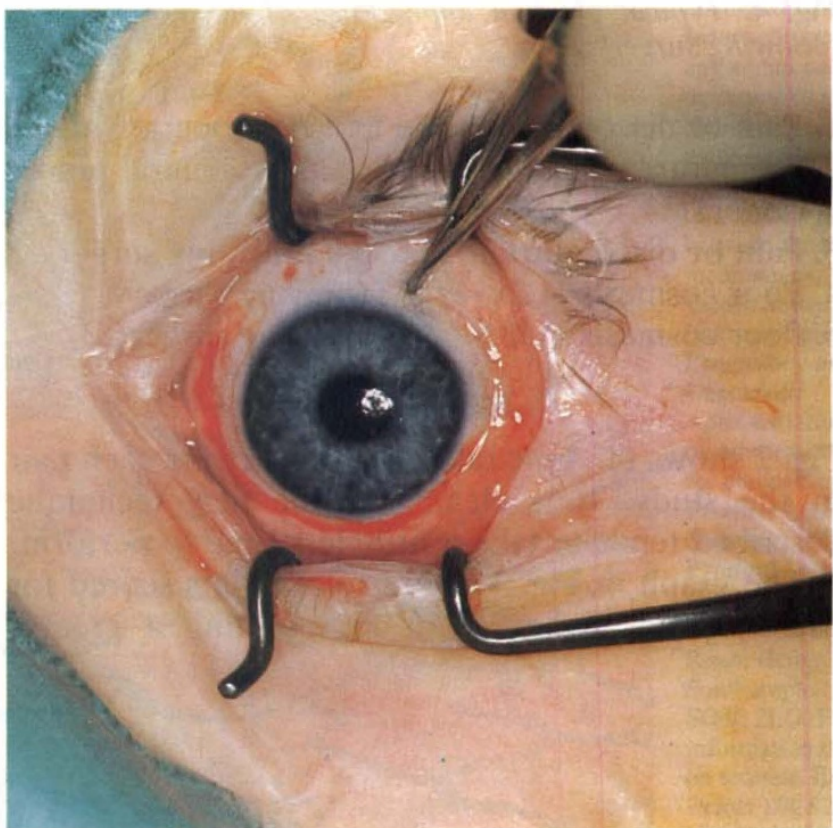

(d)

Fig. 1. (a) $A 240^{\circ}$ paralimbal incision is made inferiorly. (b) Each rectus muscle is caught from below and Tenon's capsule incised superiorly. (c) The muscle is cleaned with forceps. (d) The conjunctiva is repositioned with moist cellulose sponge.

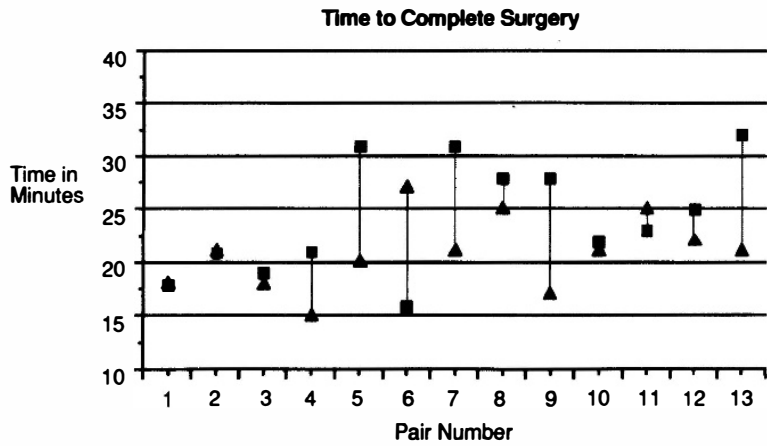

Fig. 2. Time to complete surgery for pairs of patients matched for age and surgeon. Triangles, method A; squares, method B.

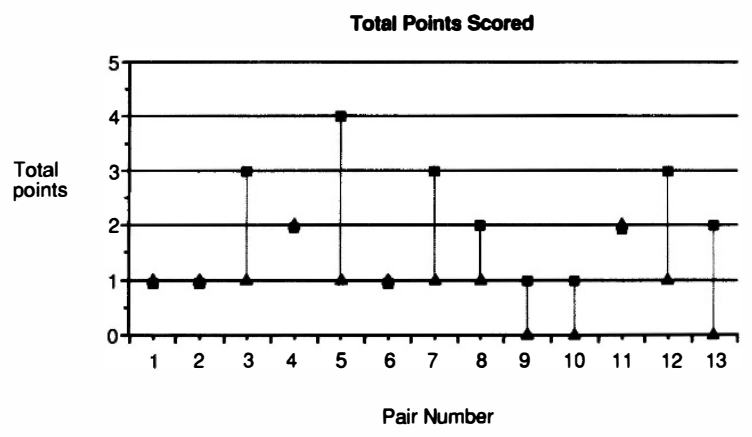

Fig. 3. Total points scored for pairs of patients matched for age and surgeon. Triangles, method A; squares, method B. 


\title{
A NOVEL CONJUNCTIVAL INCISION FOR HORIZONTAL STRABISMUS SURGERY
}

\author{
A. B. CALLEAR and E. M. EAGLING \\ Birmingham
}

\begin{abstract}
SUMMARY
We present a randomised, controlled study of a new approach to conjunctival incision in horizontal strabismus surgery. A peritomy is performed from 2 to 10 o'clock inferiorly allowing free access to the horizontal recti from below. No limbal stay sutures are required and the conjunctiva is left unsutured on completion of surgery. Results of 13 patients are presented. Each patient is paired with a control on whom conventional surgery was performed. The pairs were matched for age and surgeon. Results show a decrease in the time taken for the procedure and decreased discomfort in the postoperative period. The external appearance of the operated eye and the incidence of complications over a 6 week period were the same for both techniques.
\end{abstract}

The traditional approach in horizontal strabismus surgery is to open the conjunctiva at two separate locations to expose the medial and lateral rectus muscles respectively. ${ }^{1,2}$ Whilst there have been reports of a number of variations on the type and position of these incisions, the authors are unaware of any description of a single limbal incision allowing access to both muscles.

There are two surveys of suture preference in strabismus surgery in the literature. ${ }^{3,4}$ Both papers report a preference for no suture to close the conjunctiva in a small minority of the surgeons polled. No further details are given.

This study attempts to evaluate a continuous paralimbal incision through $240^{\circ}$ inferiorly in comparison with a widely used conventional technique. The method under study allows easy access to the horizontal recti without the need for limbal stay sutures. Inferior oblique muscle surgery is also possible through the same incision. In addition the conjunctiva can be left unsutured on completion of

From: Birmingham and Midland Eye Hospital, Birmingham, UK.

Correspondence to: Andrew Callear, Birmingham and Midland Eye Hospital, Church Street, Birmingham B3 2NS, UK. the operation. The authors have attempted to assess the effect of this method on the time taken for surgery, post-operative discomfort and complication rates.

\section{PATIENTS AND METHODS}

Thirteen pairs of patients admitted under the care of the authors for horizontal strabismus surgery were matched for age and surgeon. Method A was used for surgery on one member of the pair, method $B$ for the other. Allocation was randomised according to a pre-prepared table.

\section{Method A}

No limbal stay suture is used. The conjunctiva is incised at the limbus through $240^{\circ}$ from 2 to 10 o'clock below. Tenon's capsule is divided inferiorly and a squint hook inserted under the horizontal rectus. The episcleral tissue is then incised with scissors above and the muscle stripped with forceps. Recession/resection of the horizontal recti is then carried out in the traditional manner. On completion of surgery the conjunctiva is repositioned with a moist cellulose sponge and the eye left unpadded (Fig. 1).

\section{Method B}

A 6.0 silk stay suture is positoned at the limbus above and below. The conjunctiva is opened separately for each horizontal rectus muscle in the conventional manner. Recession/resection is performed as above and the conjunctiva closed with interrupted 8.0 vicryl.

Both groups received topical amethocaine and chloramphenicol on completion of surgery. Operations were timed from insertion of the lid speculum to its removal. Results were compared over a 6-week period by completing a proforma.

A 5-point scale was used to assess each patient. One point was awarded for each of the following: 


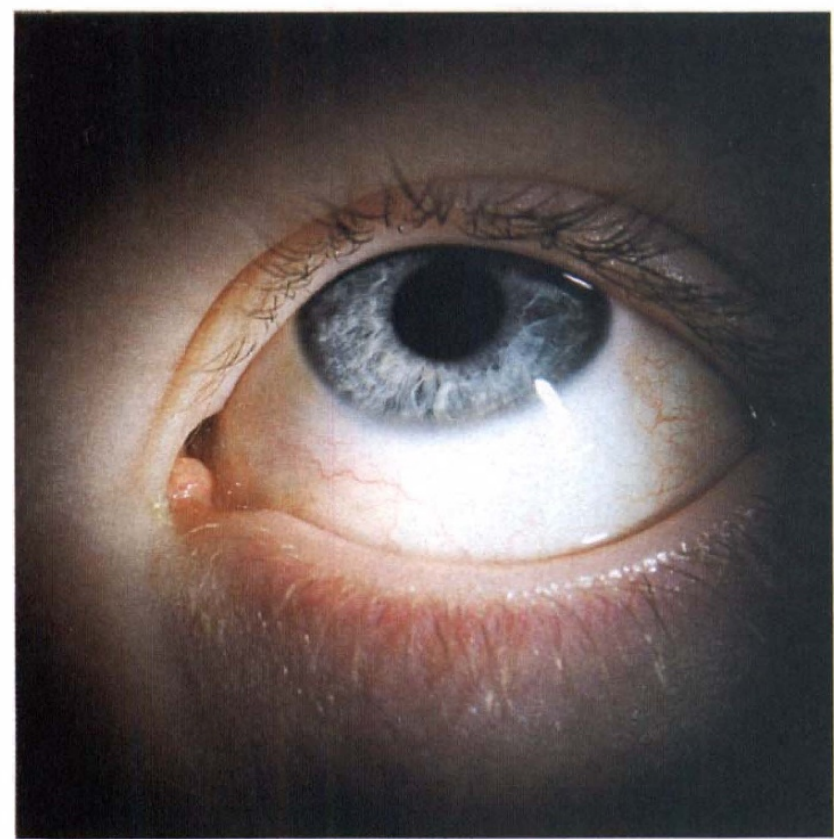

Fig. 4. Typical external appearance of the eye 6 weeks following surgery.

1. Pain or discomfort on the first post-operative day.

2. Premature return to outpatients or general practitioner.

3. Pain or discomfort at 2 weeks following surgery.

4. Poor cosmesis at 2 weeks following surgery.

5. Poor cosmesis at 6 weeks following surgery.

\section{RESULTS}

Fig. 2 shows the time taken for surgery for each pair in the study. It is clear that the new technique described tends to take a little less time to perform.

The graph in Fig. 3 shows total points scored for each pair, a higher score representing a greater incidence of complications. It can be seen that the new method gives a generally lower score. In particular there were no problems with long-term cosmesis. Fig. 4 shows the typical external appearance of the eye at 6 weeks following surgery.

\section{DISCUSSION}

This short study supports the authors' impressions that the novel conjunctival incision described above can shorten anaesthetic time and may reduce the amount of post-operative discomfort in children undergoing strabismus surgery. No increase in postoperative complications has been demonstrated and the long-term cosmetic effect appears equal to conventional methods.

The study is subject to observer bias as surgeons cannot be masked to the method used. Furthermore the qualitative nature of the data makes formal statistical analysis invalid.

In spite of this there is sufficient evidence to recommend this approach as a safe and more efficient approach to horizontal rectus muscle surgery.

Key words: Complications, Conjunctiva, Controlled, Randomised, Rectus, Stabismus surgery.

\section{REFERENCES}

1. Von Noorden GK. The limbal approach to surgery of the rectus muscles. Arch Ophthalmol 1968;80:94-7.

2. Willshaw HE. Rectus muscle surgery: how to do it. Trans Ophthalmol Soc UK 1986;105:583-8.

3. Schwartz RL, Koller HP. Survey of sutures used in strabismus surgery. J Pediatr Ophthalmol Strabismus 1981;18:39-41.

4. Dunlap EA. Survey of sutures used in strabismus surgery. Am J Ophthalmol 1972;74:625-6. 